\title{
Experiencias del uso del portafolio para residentes de la Clínica Universidad de Navarra
}

\author{
Javier Schlatter
}

Introducción. En el año 2008 se implantó el portafolio electrónico para residentes en la Clínica Universidad de Navarra. Materiales y métodos. Hemos recabado los datos mediante una herramienta informática para conocer su cumplimiento online.

Resultados. El cumplimiento es universal en los aspectos asistenciales, pero bajo en investigación y profesionalismo, sobre todo en los R1. La mitad registra las entrevistas con el tutor, mientras que éste sólo firma la mitad de las entrevistas.

Conclusiones. Tras tres años de experiencia, el cumplimiento del portafolio por el residente se puede considerar alto, y algo menor el del tutor.

Palabras clave. Educación médica. Evaluación formativa. Portafolio. Residente. Tutor.

\section{Experiences about the University Clinic of Navarra portfolio in medical education}

Introduction. In 2008, the residents of the University Clinic of Navarra began to use a new electronic portfolio.

Materials and methods. In order to know their fulfillment, we have collected the data by an online tool.

Results. The total of trainees fulfilled the clinical sections, but the data about professionalism and research were less performed, particularly by the R1's. Half of the residents fulfilled the interviews with the trainers, and these ones signed only half of these interviews.

Conclusions. After three years, the portfolio accomplishment by the residents could be considered high, and a little lower by the trainers.

Key words. Formative assessment. Medical education. Portfolio. Residents. Trainers.

\section{Introducción}

El portafolio del residente de la Clínica Universidad de Navarra (PRCUN) es una aplicación informática diseñada para que cada residente gestione, en colaboración con su tutor, su propio plan de desarrollo formativo-profesional[1,2].

La idea de desarrollar un portafolio surgió en el año 2006. Pretendíamos salvar algunas limitaciones del libro del residente, a la vez que buscábamos una herramienta que reflejara los avances y deficiencias del aprendizaje y actividad asistencial, de cara a la evaluación formativa $[3,4]$.

Durante dos años se puso en marcha un proyecto inicial e hicimos un ensayo piloto con varios residentes. Una vez hechas las principales correcciones, el PRCUN comenzó a implantarse en mayo de 2008 con los residentes recién incorporados. En la actualidad son tres las promociones de residentes que lo han incorporado.
Los fundamentos teóricos que justifican el empleo de esta herramienta son $[5,6]$ :

- Las evidencias recogidas permiten identificar competencias consolidadas y deficiencias.

- La autoobservación de la información permite al residente un análisis reflexivo y autoevaluativo que mejora su actitud proactiva ante el aprendizaje.

- La determinación por el residente de estrategias de mejora de sus déficits competenciales y de nuevas necesidades de aprendizaje, más la confirmación por el tutor, aumenta su adhesión y actitud de compromiso con los objetivos.

- Permite considerar en la evaluación no sólo las realidades formativas del residente, sino también su actitud ante el aprendizaje y mejora continua.

Las características que queríamos para el portafolio del residente eran:

- Que fuera lo más sencillo posible y supusiera el menor tiempo posible de dedicación, por ejem-
Comisión de Docencia. Clínica Universidad de Navarra. Pamplona, Navarra, España.

Correspondencia:

Dr. Javier Schlatter.

Comisión de Docencia. Clínica Universidad de Navarra. Avda. Pío XII, 36. E-31008

Pamplona (Navarra).

E-mail:

schlatter@unav.es

Equipo de trabajo del portafolio: Salvador Martín-Algarra, María de la Viesca, Elena Fernández-Jarne, María J. García-Velloso, María J. Yepes, Myriam Fernández-Alonso, Elena Faedda, Esther FernándezUrrizola, Sagrario Azpilicueta.

Agradecimientos:

A los miembros de la Comisión de Docencia y de Calidad, al Servicio de Informática de la Clínica y a todos los residentes que, con sus aportaciones y esfuerzo, hacen del portafolio un instrumento en constante proceso de mejora.

Conflicto de intereses: No declarado.

Conflict of interests: None declared.

(c) 2013 FEM 
Tabla I. Cumplimiento de los distintos apartados del portafolio de los R1-R3 y optativos.

\begin{tabular}{llll}
\hline & $\begin{array}{c}\mathrm{R} 1-\mathrm{R} 3 \\
(n=107)\end{array}$ & $\begin{array}{c}\text { Optativos } \\
(n=30)\end{array}$ & $\begin{array}{c}\text { Total } \\
(n=137)\end{array}$ \\
\hline Actividad asistencial & $107(100 \%)$ & $30(100 \%)$ & $137(100 \%)$ \\
\hline Competencias: conocimientos & $54(51 \%)$ & $6(20 \%)$ & $60(44 \%)$ \\
\hline Competencias: docencia & $51(48 \%)$ & $7(23 \%)$ & $58(42 \%)$ \\
\hline Competencias: investigación & $31(29 \%)$ & $11(37 \%)$ & $42(31 \%)$ \\
\hline Competencias: profesionalismo & $16(15 \%)$ & $3(10 \%)$ & $19(14 \%)$ \\
\hline Objetivos & $45(42 \%)$ & $4(13 \%)$ & $48(35 \%)$ \\
\hline Entrevistas formativas & $70(65 \%)$ & $15(50 \%)$ & $85(51 \%)$ \\
\hline Entrevistas formativas (firma del tutor) & $26(24 \%)$ & $2(7 \%)$ & $28(20 \%)$ \\
\hline Evaluaciones & $9(8 \%)$ & $2(7 \%)$ & $11(8 \%)$ \\
\hline
\end{tabular}

plo permitiendo que la información pudiera volcarse directamente desde el sistema informático de asistencia de CUN (SICUN), lo que añadiría además fiabilidad a esos datos.

- Que sólo hubiera un sitio tanto para la recogida de información como para la revisión por el tutor.

- Que fuera universal, aplicable a todos los residentes aunque se pudieran hacer especificaciones para algún departamento o especialidad.

- Que incluyera toda la información necesaria para la evaluación formativa y todo lo que el residente quisiera que constara para que su esfuerzo quedara suficientemente explicitado; por esto interesaría que fuera en algunos aspectos irrestricto.

- Que respetara la confidencialidad (acceso mediante clave), aunque también pudiera acceder su tutor y el vocal correspondiente de la Comisión de Docencia.

- Que permitiera la confirmación por el tutor de la información que vuelca el residente, en un clima a la vez de confianza, pues no toda la información podría ser respaldada por un documento adjunto.

Teniendo en cuenta estos fundamentos teóricos y características básicas desarrollamos el PRCUN, que actualmente consta de los siguientes apartados:

- ¿Qué es el portafolio?: incluye un documento teórico y otro práctico sobre modo de uso.

- Datos del residente: datos de identificación y contacto, plan de rotaciones previsto o realizado e historial de accesos al SICUN (este último de forma automática).

- Documentación: documentos personales del residente, los oficiales que le afectan, así como impresos de utilidad.

- Objetivos profesionales: lo cumplimenta el residente cuantas veces desee y recoge los logros alcanzados desde la vez anterior y los objetivos para el nuevo periodo, permitiendo un comentario y conformidad del tutor.

- Confirmación de competencias profesionales: el tutor declara su competencia inicial como residente.

- Competencias: se subdivide en conocimientos (cursos, sesiones recibidas...), investigación (tesis, ponencias, publicaciones...), docencia (sesiones impartidas...) y profesionalismo (trabajo en equipo y orientado a las personas, capacidad proactiva, comunicación interpersonal, y control emocional). Incluye un apartado para añadir aspectos no reglados pero que suponen un enriquecimiento en su aprendizaje.

- Formación obligatoria: participación en actividades preceptivas como los cursos de reanimación cardiopulmonar, de protección radiológica, etc.

- Entrevistas formativas: recoge un resumen de las entrevistas periódicas del residente con el tutor y otros profesionales implicados en su formación.

- Guardias: con las fechas y tipo de guardias realizadas, y un espacio para comentarios.

- Actividad asistencial: muestra la asistencia realizada (notas de evaluación, informes médicos...).

- Evaluaciones: se anotan opcionalmente las evaluaciones sumativas (rotaciones, anuales, board...).

- Currículo: permite el acceso a la web Universia XXI para redactar su currículo, e incorporar los documentos originales necesarios.

- Notas del residente: anotaciones a las que sólo tiene acceso el residente; en el acceso que tiene el tutor hay también un apartado similar restringido para él.

- Visado: lo cumplimenta el tutor al menos una vez al año, para dejar constancia de su supervisión.

- Informes de resumen de rotaciones: permite al residente reflexionar sobre una rotación, aprendizaje recibido y puntos futuros de mejora.

- Informes de resumen de competencias: el residente reflexiona sobre la adquisición de un determinado aspecto competencial con propuesta de modos de mejora.

Todas las hojas del PRCUN muestran un calendario donde resaltan las fechas de entrevistas y guardias. Para la confirmación del tutor se utiliza un 
Tabla II. Cumplimiento de los distintos apartados del portafolio del residente por año de residencia.

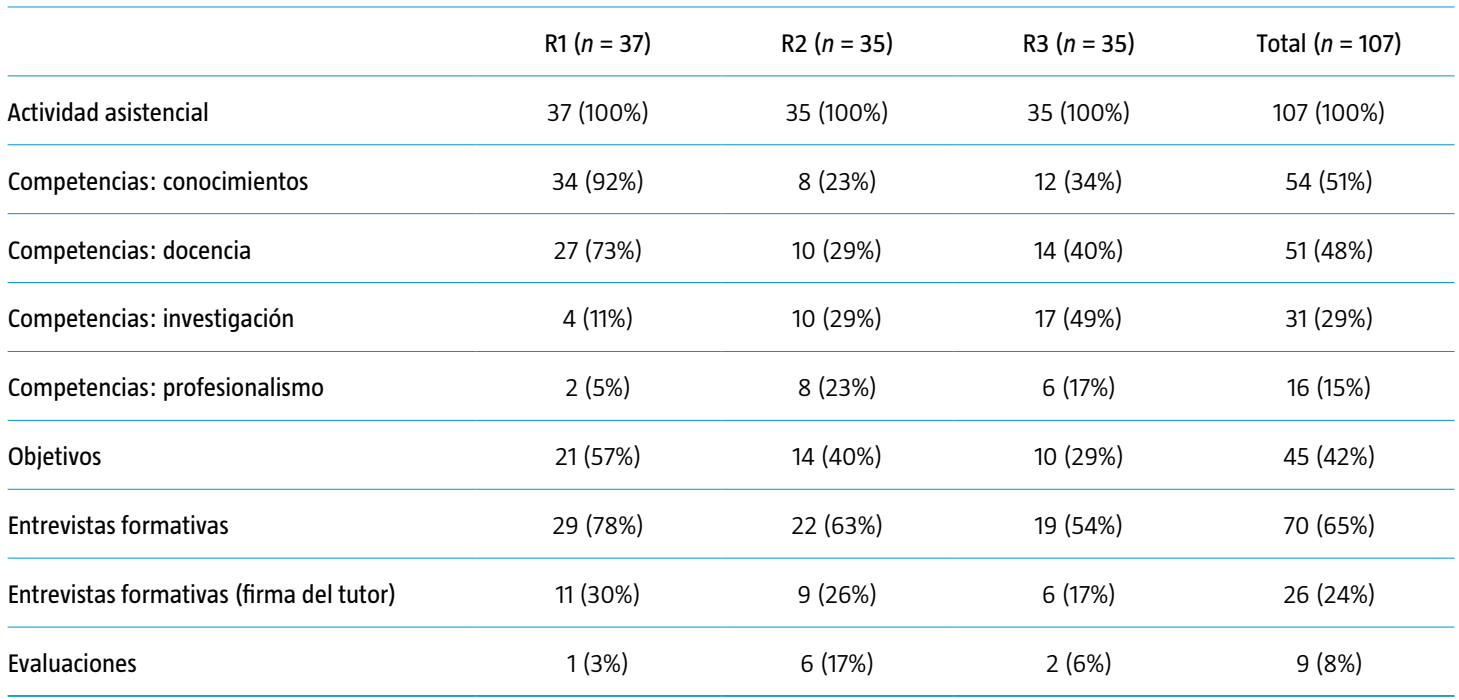

código de señales tipo semáforo (roja si no está confirmada por ninguno de los dos, amarilla cuando la ha confirmado sólo el residente, y verde cuando ambos la han confirmado). En la barra superior se encuentra la aplicación para guardar documentos mediante grabado o escaneado.

Los objetivos de este trabajo son evaluar el cumplimiento de los residentes y tutores del PRCUN en sus distintos apartados y reflexionar sobre las causas que subyacen en esos datos.

\section{Materiales y métodos}

Desde que comenzamos a utilizar el PRCUN son tres las promociones que lo han incorporado, lo que supone un total de 130 residentes. De ellos, hay algunos departamentos de especialidades básicas que o bien cumplimentan el libro editado por la comisión nacional de su especialidad (como radiofarmacia), o no tienen aún adaptado el portafolio al SICUN en la parte asistencial, por lo que algunos utilizan también el libro del residente. De este modo, en vez de 130, el número de residentes que deberían estar empleando el PRCUN son 107. Por otra parte, desde el inicio, ofrecimos a otros residentes de años superiores incorporar esta herramienta; lo han hecho 30, por lo que el número total de quienes lo están utilizando es de 137.

Para evaluar la adhesión de los residentes al portafolio utilizamos una herramienta del SICUN que permite conocer online qué apartados se han cum- plimentado, en este caso entre el 1 de mayo y el 1 de noviembre de 2010.

\section{Resultados}

El dato más relevante es que la totalidad (100\%) de los residentes que tienen previsto emplearlo - tanto R1 a R3, como el grupo de opcionales- lo han usado en estos meses.

En esta misma línea, el 100\% lo han empleado al menos una vez para registrar su actividad asistencial (Tabla I).

En cuanto a las distintas competencias, las más cumplimentadas son las de conocimientos y docencia, que han rellenado el $44 \%$ y $42 \%$, respectivamente, del global; en menor proporción, la referente a investigación (31\%), y muy por debajo, la de profesionalismo (14\%). Si tenemos en cuenta el año de residencia (Tabla II), el porcentaje en conocimientos y docencia alcanza hasta un $92 \%$ y $73 \%$, respectivamente, en los R1, mientras que en investigación, en cambio, el porcentaje va en descenso del R3 al R1 (49\%, $29 \%$ y $11 \%$, respectivamente). El apartado de profesionalismo es poco utilizado en todos los años.

Las entrevistas formativas las han registrado un $51 \%$ del total, aunque asciende al $65 \%$ en el grupo R1-R3. Divididos por años de residencia, el porcentaje es mayor en los R1 (R1: 78\%; R2: 63\%; R3: 54\%). El apartado de objetivos sigue una tendencia similar, aunque las cifras son inferiores tanto el total (35\%), como por años (R1: 57\%; R2: 40\%; R3: 29\%). 
Un dato interesante es la firma del tutor en las entrevistas formativas. El porcentaje de tutores que han firmado las entrevistas es del 20\% (frente al 51\% de entrevistas registradas). Analizado por años, aunque en los $\mathrm{R} 1$ asciende al 30\%, la comparación frente al 78\% de entrevistas registradas sigue siendo baja.

Por último, el apartado de evaluaciones muestra los resultados más bajos (8\%), con un porcentaje similar en la media de los R1-R3.

\section{Discusión}

El PRCUN es una aplicación informática diseñada para que cada residente gestione, en colaboración con su tutor, su propio plan de desarrollo profesional.

El hecho de que el 100\% de los residentes candidatos a utilizarlo en estas tres primeras promociones lo hayan empleado entendemos que sugiere una valoración positiva. Además, los porcentajes han aumentado en las sucesivas promociones, lo que orienta también a un proceso de implantación progresiva de la herramienta.

Todos los residentes implicados sin excepción han registrado y valorado su actividad asistencial al menos una vez en estos meses. Entendemos que influye positivamente el fácil acceso a la información.

Dentro de los apartados de competencias, aunque hay un cumplimiento mayor de los R1 en general, en el apartado de investigación son los R3 y los opcionales de años superiores los que más lo han registrado, probablemente por su mayor interés en los años finales de residencia. Por último, el apartado de profesionalismo ha sido el menos cumplimentado; es probable que el interés del residente sea menor, pero también nos ha hecho replantear la simplificación del cuestionario utilizado [7].

El porcentaje de entrevistas formativas, aunque superior al $50 \%$, pensamos que no se corresponde con la realidad, según los datos de las encuestas anuales a R2 y R4, donde esas cifras son superiores. En este apartado, donde hay que describir y resumir, es posible que se pierda de hecho información. Llama más la atención el bajo porcentaje de firmas del tutor, por lo que supone de baja adhesión en un aspecto tan crucial de la relación docente tutor-residente [8]. El comentario sobre el apartado de objetivos es similar, pero acentuado si tenemos en cuenta que el porcentaje de cumplimiento es menor. Por último, el apartado de evaluaciones no pa- rece útil para el residente, probablemente porque prefieren registrarlo en el currículo o porque lo ven poco relevante.

No hemos podido obtener los datos del currículo por problemas del programa informático. Sí sabemos que los residentes de años superiores, con más bagaje de investigación, están empleando el enlace al programa web Universia XXI, mientras que los R1 y R2 hacen su currículo al margen del portafolio.

Recientemente hemos añadido los dos apartados de informes de resumen, tanto de rotaciones como de competencias, por lo que aún no tenemos información consistente. Estos apartados tendrán una gran importancia en el proceso de aprendizaje reflexivo y autocrítico del residente, y en el diseño y desarrollo de su itinerario formativo.

Por tanto, y pese a su reciente implantación, pensamos que los resultados orientan a una buena acogida por los residentes y algo menor por parte de los tutores. Las frecuentes sugerencias y aportaciones de unos y otros están permitiendo corregir, y especialmente simplificar, una herramienta que debe aunar eficacia y sencillez en su manejo si quiere cumplir su objetivo. A la espera de que exista una herramienta similar en el ámbito nacional, parece de momento un avance y una contribución interesante en el proceso formativo del residente y en la tarea docente del tutor.

\section{Bibliografía}

1. Wade RC, Yarbrough DB. Portfolios: a tool for reflective thinking in teacher education? Teach Teacher Educ 1996; 12: 63-79.

2. Soler-Durall C. Reflexiones sobre el hospital: su función en la formación de médicos. Educ Med 2010; 13: 71-5.

3. Dannefer EF, Henson LC. The portfolio approach to competency-based assessment at the Cleveland Clinic Lerner College of Medicine. Acad Med 2007; 82: 493-502.

4. Canalejas-Pérez MC. El portafolio como herramienta didáctica: un estudio en escuelas universitarias de enfermería. Educ Med 2010; 13: 53-61.

5. Imhof M, Picard C. Views on using portfolio in teacher education. Teach Teacher Educ 2009; 25: 149-54.

6. Institut d'Estudis de la Salut. Portafolio SEMI (Sociedad Española de Medicina Interna). Guía de trabajo. Barcelona: Generalitat de Catalunya; 2007.

7. Kalet AL, Sanger J, Chase J, Keller A, Schwartz MD, Fishman ML, et al. Promoting professionalism through an online professional development portfolio: successes, joys, and frustrations. Acad Med 2007; 82: 1065-72.

8. Ruiz R. Opiniones de tutores y residentes tras aplicar un sistema de evaluación formativa tipo portafolio: la nueva propuesta de 'guía de práctica reflexiva' del libro del especialista en medicina de familia en formación. Educ Med 2008; 11: 147-55. 